\title{
Declínio cognitivo, depressão e fragilidade em idosos: incidência e relações
}

\author{
Cognitive decline, depression and frailty in the elderly:
} incidence and relations

Deterioro cognitivo, depresión y fragilidad en los ancianos: incidencia y relaciones

\author{
Aléxia Ximenes Torqueti \\ Edvaldo Soares
}

RESUMO: O objetivo da pesquisa foi verificar a incidência de declínio cognitivo, depressão e fragilidade em amostra composta por idosos institucionalizados e idosos residentes na comunidade e, a partir disso, identificar correlações entre essas variáveis. Foram sujeitos da pesquisa 33 idosos residentes em duas ILPI's e idosos da comunidade atendidos no Centro de Estudos da Educação e da Saúde, cidade de Marília, SP. Para coleta de dados relativos ao declínio cognitivo, foram utilizados o MEEM e o CDR; o GDS-30 para identificar a incidência de depressão e o EFS, para a fragilidade. Para a análise de correlação entre as variáveis, foi utilizado o coeficiente de correlação de Pearson e o coeficiente de correlação de Spearmann por restrição teórica. Idosos institucionalizados se mostraram mais vulneráveis em relação a todas as variáveis analisadas. A depressão está positivamente correlacionada com a fragilidade e com a capacidade cognitiva. A fragilidade influencia de maneira positiva a capacidade cognitiva. Os resultados da análise indicaram correlação regular negativa entre o MEEM e o CDR. A identificação dos fatores de risco foram relacionados ao declínio cognitivo e às demências; à depressão e à fragilidade, bem como as relações entre tais fatores são fundamentais para o estabelecimento de estratégias preventivas e terapêuticas.

Palavras-chave: Idoso; Transtornos cognitivos; Depressão; Fragilidade; Prevalência. 
ABSTRACT: The objective of the research was to verify the incidence of cognitive decline, depression and frailty in a sample composed of institutionalized elderly people and elderly residents in the community and, from this, to identify correlations between these variables. The study subjects were 33 elderly people living in two Long-term Institutions for Elderly People (LIEP) and the elderly of the community that were attended at the Center for Education and Health Studies, city of Marilia, SP. In order to collect data on cognitive decline, the Mini Mental Status Exam (MMSE) and CDR were used; GDS-30 to identify incidence of depression and EFS for frailty. For the correlation analysis between the variables, the Pearson correlation coefficient and Spearmann correlation coefficient were used due to theoretical restriction. Institutionalized elderly individuals were more vulnerable in relation to all analyzed variables. Depression is positively correlated to frailty and cognitive ability. Fragility positively influences cognitive ability. The results of the analysis indicated a negative regular correlation between MMSE and CDR. The identification of risk factors related to cognitive decline and dementias; to depression and frailty, as well as the relations between these factors are fundamental for the establishment of preventive and therapeutic strategies.

Keywords: Elderly; Cognitive disorders; Depression; Frailty; Prevalence.

RESUMEN: El objetivo de esta investigación fue verificar la incidencia de deterioro cognitivo, depresión y fragilidad en una muestra compuesta por ancianos institucionalizados y ancianos que viven en la comunidad, y de esto, identificar correlaciones entre estas variables. Treinta y tres residentes mayores de dos LSIE y personas mayores que viven en la comunidad asistieron al Centro de Estudios de Educación y Salud, ciudad de Marília, SP, fueron encuestados. Para la recopilación de datos relacionados con el deterioro cognitivo, se utilizaron MMSE y CDR; GDS30 para identificar la incidencia de depresión y EFS por fragilidad. Para el análisis de correlación entre las variables, utilizamos el coeficiente de correlación de Pearson y el coeficiente de correlación de Spearmann por restricción teórica. Los ancianos institucionalizados fueron más vulnerables en relación con todas las variables analizadas. La depresión se correlaciona positivamente con la fragilidad y la capacidad cognitiva. La fragilidad influye positivamente en la capacidad cognitiva. Los resultados del análisis indicaron una correlación regular negativa entre MMSE y CDR. La identificación de los factores de riesgo se relacionó con el deterioro cognitivo y la demencia; la depresión y la fragilidad, así como las relaciones entre dichos factores son fundamentales para el establecimiento de estrategias preventivas y terapéuticas.

Palabras clave: Ancianos; Trastornos cognitivos; Depresión; Fragilidad; Prevalencia. 


\section{Introdução}

O envelhecimento é um evento natural e progressivo. Pode ser avaliado em função da diminuição da capacidade de suportar sobrecargas do ambiente. Diversas variáveis endógenas e exógenas estão envolvidas, de forma complexa e integrada, na diminuição da capacidade de suportar as sobrecargas do ambiente. Entre essas variáveis, poderíamos citar as alterações psicológicas e as alterações fisiológicas, as quais são, por sua vez, também condicionadas por fatores genéticos e epigenéticos.

Essas alterações podem ser abordadas, de maneira não reducionista, a partir da perspectiva do comprometimento no funcionamento dos sistemas do organismo, entre os quais, do Sistema Nervoso, que tem entre seus papéis, conforme perspectiva de Claude Bernard (1813-1878), célebre professor e pesquisador do Collège de France, o de coordenar as funções vitais do organismo, mantendo, assim, a integridade e unidade do mesmo (Bernard, 1878/1996)

O Sistema Nervoso é um dos sistemas mais afetados no/pelo processo de envelhecimento. Modificações relacionadas à hipotrofia encefálica e alterações no sistema de neurotransmissores estão relacionadas a prejuízos cognitivos, transtornos de humor e diminuição da capacidade funcional. Diminuição da capacidade funcional, declínio da capacidade cognitiva e transtornos de ordem psiquiátrica são fatores diretamente ligados à perda da autonomia do indivíduo (Cruz, et al., 2015; Rabelo, 2009). Prejuízos cognitivos podem estar relacionados a diversos fatores, entre os quais, a idade.

A literatura indica que o declínio cognitivo progressivo está implicado no desenvolvimento de demências (Laks, Rozenghal, \& Engelhardt, 1995). Tal fato é preocupante, considerando-se a alta taxa de prevalência de declínio cognitivo entre as populações idosas no Brasil (Gurian, et al., 2012; Herrera, Caramelli, \& Nitrini, 1997) e no exterior (Aevarsson, \& Skoog, 1997; Graves, et al., 1996), com destaque para a maior prevalência de declínio cognitivo entre os idosos institucionalizados (Trindade, et al., 2013). Interessante destacar que, entre os fatores protetores ao declínio cognitivo, está, além dos cuidados com a saúde, como por exemplo, o combate à hipertensão arterial (Duron, \& Hanon, 2008), a educação e a manutenção de atividades mentais (Soares, Coelho, \& Carvalho, 2012; Hallet, et al., 2009). 
Entre os transtornos de humor mais prevalentes em populações idosas está a depressão, a qual apresenta prevalência que varia entre $13 \%$ e $14 \%$, entre idosos residentes em comunidade (Siqueira, 2009; Lindesay, Briggs, \& Murphy, 1989; Gurland, et al., 1978). Essa taxa aumenta de forma significativa entre os idosos institucionalizados, chegando ao índice de 50\% (Siqueira, 2009; Heiser, 2004). A literatura também tem indicado que, entre os indivíduos do sexo feminino, a prevalência de episódios depressivos é mais recorrente (Bertoldi, Batista, \& Ruzanowsky, 2015; Kockler, \& Heun, 2002; Angst et al., 2002). Estudos relativos à relação entre depressão e áreas cerebrais têm destacado alterações nas áreas frontais e suas conexões, bem como nas áreas temporais (Rozenthal, Laks, \& Engelhardt, 2004). Cabe ressaltar que os lobos frontais e temporais estão envolvidos em tarefas cognitivas, tais como a atenção e a memória. Há evidências clínicas suficientes que apontam para a depressão como um fator de risco para declínio cognitivo e demência (Steffens, \& Potter, 2008; Steffens, et al., 2006; Jorm, 2000), bem como para a fragilidade (Ní Mhaoláin, et al., 2012), aqui entendida como uma síndrome multidimensional, que envolve vários fatores biológicos, físicos, cognitivos, sociais, econômicos e ambientais, e que pode se manifestar em indivíduos de todas as faixas etárias (Rolfson, et al., 2006). Também é importante destacar o fato de que alguns déficits cognitivos, decorrentes de episódios depressivos, podem persistir após a remissão clínica (Trichard, et al., 1995).

A fragilidade em indivíduos idosos é apontada por Gobbens e colaboradores (2010) como sendo resultado da diminuição da reserva fisiológica e da capacidade de manutenção da homeostase, o que aumenta o risco de vulnerabilidade às sobrecargas do ambiente. As taxas de prevalência de fragilidade variam de 5,8\% a 27,3\%, em países da Europa e da América do Norte, com maior incidência em idosos institucionalizados e mais idosos (Santos-Eggimann, et al., 2009; Rockwood, et al., 2004). Em países em desenvolvimento, as taxas variam de 26,7\% a 42,6\% (Soares, Cruz, \& Carvalho, 2016; Sousa, et al., 2012). Pesquisas apontam a fragilidade como fator de risco para o declínio cognitivo e para o desenvolvimento de episódios depressivos (John, \& Tyas, 2013; Rockwood, Abeysundera, \& Mitnitski, 2007).

$\mathrm{Na}$ literatura científica, os estudos sobre a fragilidade são recentes, e pesquisas que buscam compreender e aprofundar as correlações entre fragilidade, declínio cognitivo e depressão são escassas. A compreensão e o aprofundamento das relações entre declínio cognitivo, depressão e fragilidade se mostram um desafio, face à multiplicidade de variáveis envolvidas. Contudo, tal compreensão e aprofundamento podem contribuir para o desenvolvimento de estratégias mais eficientes de intervenções na área da saúde pública. 
O objetivo deste estudo foi verificar a incidência de declínio cognitivo, depressão e fragilidade em uma amostra composta por idosos institucionalizados e idosos residentes em comunidade e, a partir disso, identificar possíveis correlações entre essas variáveis.

\section{Métodos e Procedimentos}

Esta pesquisa se trata de um estudo descritivo, analítico e de corte transversal. O levantamento da amostra foi realizado em duas Instituições de Longa Permanência para Idosos (ILPI's) localizadas na cidade de Marília, SP, e no Centro de Estudos da Educação e da Saúde, CEES, UNESP - Marília, SP; estudo que tem entre seus objetivos o de prestar serviços diagnósticos e intervenção nas áreas de educação, fisioterapia, fonoaudiologia e terapia ocupacional à comunidade. A coleta de dados ocorreu entre os meses de julho e novembro de 2016. Foram sujeitos da pesquisa 33 idosos, de ambos os sexos, com idade de 60 e mais anos, residentes em duas ILPI's, e idosos da comunidade atendidos no CEES. Foram excluídos da pesquisa indivíduos que apresentaram limitações cognitivas que os impediam de responder aos instrumentos e os que se negaram a participar da pesquisa.

Os dados relativos à cognição, declínio cognitivo/demência, depressão, fragilidade foram obtidos a partir da aplicação dos seguintes instrumentos:

1) Mini-Exame do Estado Mental - MEEM: utilizado para avaliar a capacidade cognitiva global (Folstein, Folstein, \& Mchugh, 1975), foi aplicado neste estudo com o propósito de avaliar a prevalência de declínio cognitivo. $\mathrm{O}$ instrumento contém questões agrupadas em sete categorias: orientação temporal (5 pontos), orientação espacial (5 pontos), registro de três palavras ( 3 pontos), atenção e cálculo ( 5 pontos), recordação das três palavras (3 pontos), linguagem (8 pontos) e capacidade construtiva visual (1 ponto). Os pontos de corte utilizados para identificação de declínio cognitivo foram de 24 pontos para indivíduos altamente escolarizados, 18 pontos para indivíduos com o ginásio (fundamental) completo e, 14 pontos para os analfabetos (Almeida, 1998).

2) Clinical Dementia Rating - CDR: concebido para avaliar a cognição e o comportamento, bem como a influência das perdas cognitivas na capacidade de realizar adequadamente as atividades de vida diária (AVD’s) (Hughes, et al., 1982). Neste estudo, foi utilizado para determinar a prevalência de demência/declínio cognitivo. 
O CDR está dividido em seis categorias cognitivo-comportamentais: memória, orientação, julgamento ou solução de problemas, relações comunitárias, atividades no lar ou de lazer e cuidados pessoais. Cada uma dessas seis categorias foi classificada conforme a pontuação: 0 = nenhuma alteração; 0,5 = questionável; $1=$ demência leve; 2 = demência moderada e, 3 = demência grave, exceto a categoria cuidados pessoais, que não possui nível 0,5 (Montaño, \& Ramos, 2005). A classificação final do CDR foi obtida pela análise dessas classificações por categorias, seguindo um conjunto de regras elaboradas e validadas por Morris (Morris, 1993). A categoria memória foi, conforme sugerida pela literatura especializada, considerada principal (Bertolucci, et al., 2001; Hughes, et al., 1982).

3) Escala de Depressão Geriátrica - GDS-30: aplicada com o objetivo de verificar sintomas depressivos em idosos (Yesavage, \& Sheikh, 1986). O escore total é dado pela somatória das respostas indicativas de depressão. Nesta pesquisa foi utilizada a interpretação de Hernandéz e Mocetzuma (1999), os quais utilizam a seguinte classificação: sem depressão = GDS até 10 pontos; com provável depressão leve = GDS de 11 a 20 pontos; e depressão moderada ou severa $=$ GDS de 21 a 30 pontos. A utilização dos pontos de corte de 10 pontos levou em consideração o critério do CID-10, conforme o qual, para o diagnóstico de depressão, o ponto de corte de 10 apresenta sensibilidade de $100 \%$ e especificidade de 88\%, (Stoppe Júnior, Jacob Filho, \& Louzã Neto, 1994).

4) Edmonton Frail Scale - EFS: escala utilizada para avaliar a fragilidade na população idosa. Avalia nove domínios e onze itens, conforme a seguinte pontuação: cognição (Teste do Desenho de um Relógio - TDR = 2 pontos; estado de saúde geral (número de internações no último ano = dois pontos); e descrição da saúde = 2 pontos; independência funcional (necessidade de ajuda para 8 atividades da vida diária) = 2 pontos; suporte social (poder contar com a ajuda de alguém para atender as necessidades) $=2$ pontos; uso de medicamentos (uso de cinco ou mais medicamentos prescritos) = 1 ponto; se esquece de tomar remédio $=1$ ponto; nutrição (perda de peso recente) $=1$ ponto; humor (se sente deprimido com frequência $)=1$ ponto; continência $($ perda de controle de urina) $=1$ ponto e, desempenho funcional (teste "levante e ande" cronometrado) $=1$ ponto. A pontuação máxima é de 17 pontos, o que representa o nível mais elevado de fragilidade (Rolfson, et al., 2006). Os escores para análise da fragilidade são: 0-4 não apresenta fragilidade, 5-6 aparentemente vulnerável, 7-8 fragilidade leve, 9-10 fragilidade moderada, 11 ou mais fragilidade grave (Fabrício-Wehbe, et al., 2009). 
Utilizou-se o teste de Kolmogorov-Smirnov com correção de Lilliefors ( $\mathrm{n} \geq 30$ ) para a verificação da normalidade dos dados obtidos por meio dos instrumentos MEEM, CDR, GDS30 e EFS e Idade. Para a verificação da normalidade dos dados obtidos por meio dos instrumentos MEEM, CDR, GDS30 e EFS e Idade, foi utilizado o Coeficiente de Correlação de Pearson para distribuição de dados normais e o coeficiente de correlação de Spearmann, por restrição teórica, quando ao menos uma das distribuições dos dados foi identificada como não normal. Os critérios para a avaliação qualitativa das correlações foram: i) nula: $r_{S}=0$; ii) fraca: $0<$ I $r_{S}$ I $<0,3$; iii) regular: $0,3 \leq$ I $r_{S}$ I $<0,6$; iv) forte: $0,6 \leq$ I $r_{S}$ I $<0,9$; v) muito forte: 0,9 $\leq \mathrm{I} \mathrm{r}_{\mathrm{S}} \mathrm{I}<1 \mathrm{e}$ vi) perfeita: I $\mathrm{r}_{\mathrm{S}} \mathrm{I}=1$ (Callegari-Jacques, 2003). Adotou-se, o nível de significância de 5,0\% de probabilidade para a rejeição da hipótese nula em todos os testes e na avaliação das correlações.

A pesquisa fez parte das atividades desenvolvidas no projeto "Memória e Envelhecimento Humano", aprovado pelo Comitê de Ética e Pesquisa da Faculdade de Filosofia e Ciências, FFC, UNESP, Campus de Marília, SP (Processo 2322/2006).

\section{Resultados}

A amostra foi composta por 33 idosos, dos quais $20(60,6 \%)$ são pertencentes a duas ILPI's localizadas na cidade de Marília, SP; e 13 (39,4\%) são residentes da comunidade e atendidos pela CEES, UNESP, Marília, SP. Entre os idosos institucionalizados, 13 eram do sexo masculino e sete do feminino, ao passo que, entre os atendidos no CEES, quatro eram do sexo masculino e nove do feminino. Do total da amostra, 51,5\% eram do sexo masculino e $48,5 \%$ do feminino. A média de idade entre os sujeitos da pesquisa foi de 71,1 anos. Entre os homens, a média foi de 69,9 anos e, entre as mulheres, 73,3 anos. No tocante à idade-origem, os não-institucionalizados apresentaram média de idade menor, inclusive em relação às mulheres. Nos sujeitos institucionalizados, a média foi de 70,5 anos para os indivíduos do sexo masculino e 82,4, para o feminino; entre os residentes da comunidade: homens, 68 anos e mulheres, 64,4 anos.

Quanto à escolaridade, observou-se que $81,8 \%$ dos idosos eram alfabetizados e 18,2\% eram analfabetos. Entre os alfabetizados, 59,2 \% era do sexo masculino e 40,8\%, do feminino. A maioria dos idosos institucionalizados $(71,4 \%)$ era alfabetizada. Todos os sujeitos residentes na comunidade eram alfabetizados. A avaliação da capacidade cognitiva (MEEM), considerando-se os pontos de corte utilizados neste estudo (24/altamente escolarizados; 18/fundamental completo; 14/analfabetos) identificou-se declínio cognitivo 
em apenas dois idosos institucionalizados $(6,06 \%$ da amostra geral). A média geral dos escores foi de 26,3 pontos. Entre os sujeitos do sexo masculino, a média foi de 27,4 pontos e, entre os do sexo feminino, 25,2 pontos. A média geral de pontuação no MEEM foi maior entre os residentes na comunidade (28,3 pontos), do que entre os institucionalizados (25 pontos). Os alfabetizados apresentaram melhor desempenho (MEEM = 27,4 pontos), se comparados aos analfabetos (MEEM=21,7 pontos).

Por outro lado, a avaliação da cognição e comportamento e a influência das perdas cognitivas na capacidade de realizar adequadamente AVD's, mediante o CDR, indicou que: 29,2\% dos sujeitos apresentavam demência leve, dos quais 3 institucionalizados e 5 não institucionalizados; 27,3\%, demência questionável (5/institucionalizados e 3/comunidade); $21,2 \%$, demência moderada (6/institucionalizados e 1/comunidade); $6 \%$, demência grave (2/institucionalizados) e 21,2\% não apresentaram alteração. A média dos escores/CDR para o sexo masculino foi de 1,2 e, para o sexo feminino, de 1,1. Em relação a idosos institucionalizados, a média/CDR foi de 1,4 e, para idosos da comunidade, de 0,8; alfabetizados, média/CDR $=1$ e média/ $\mathrm{CDR}=1,8$, para os idosos analfabetos. Foi observado que os indivíduos que se mostraram sem alteração no CDR obtiveram média/MEEM de 24 pontos; os que apresentaram alterações questionáveis, 27,4 pontos/média/MEEM; demência leve, 28,2 pontos/média/MEEM; demência moderada, 18,7 pontos/média/MEEM e demência grave, 20/pontos/média/MEEM. Os resultados e tendências para MEEM e CDR podem ser observados na Figura 1.

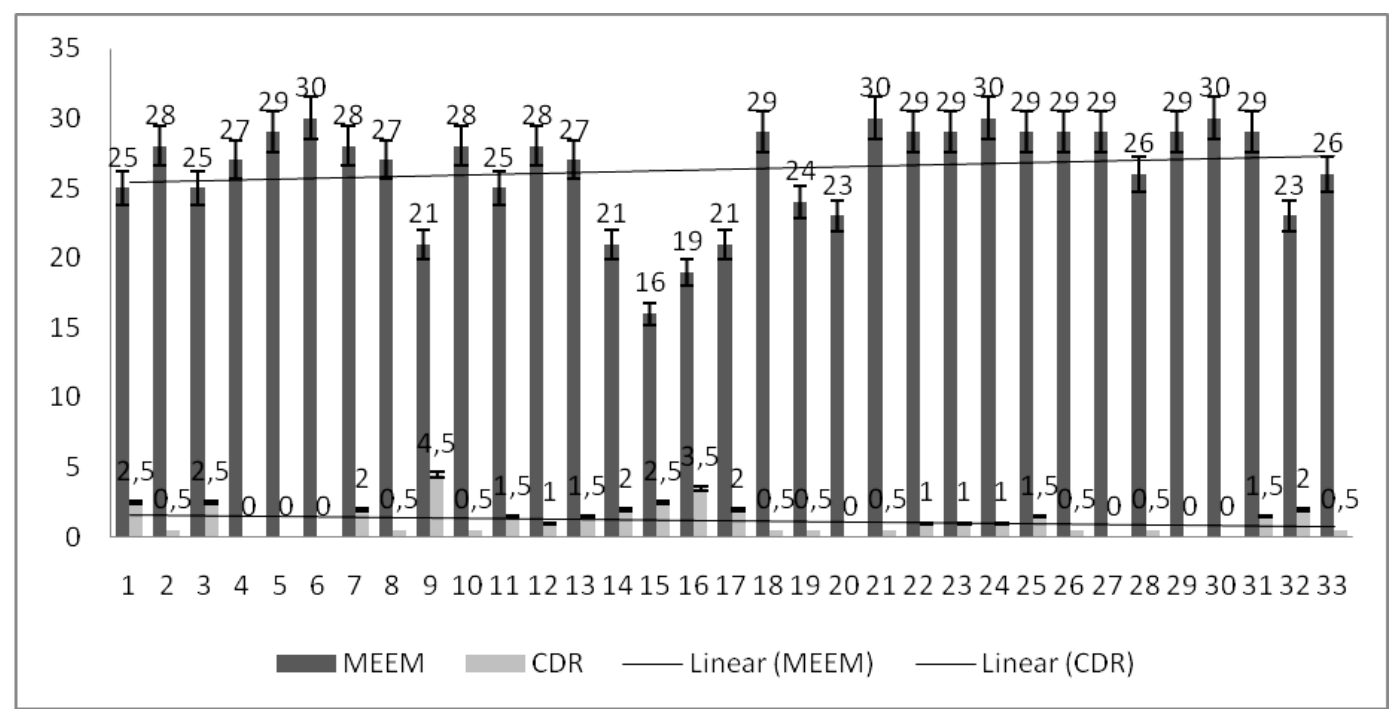

Figura 1: Desempenho individual e tendências no MEEM e CDR de 33 idosos da cidade de Marília, SP, 2016 
A avaliação da prevalência de depressão, mediante aplicação e critérios da GDS-30, apontou que $72,2 \%$ dos sujeitos avaliados não apresentaram indicativos para a depressão. E que $27,2 \%$ dos idosos apresentaram indicativo de depressão leve. Não foram identificados idosos com indicativo de depressão moderada a severa. Considerando-se o total de sujeitos que apresentaram indicativo de depressão leve $(n=9), 66,7 \%$ eram institucionalizados e, $33,3 \%$, residentes na comunidade. $55,5 \%$ dos sujeitos que apresentaram indicativo de depressão eram do sexo feminino. A média dos escores/GDS-30 para idosos do sexo masculino foi de 7,4; e 9,5 para sexo feminino. Em relação aos idosos institucionalizados, a média foi 8,8 e, para os residentes, 7,9; alfabetizados tiveram média/GDS-30 de 8,2 e analfabetos, 8,9 média/GDS-30.

Foi constatado ainda que, entre os indivíduos que apresentaram indicativo de depressão leve (n=9), a média/MEEM foi de 23,9 pontos (sem indicativo de declínio cognitivo); e a média/CDR = 1,72 pontos (tendência de demência leve a moderada).

Em termos de prevalência de fragilidade, avaliada mediante a EFS, foi observado que $72,7 \%$ dos sujeitos da pesquisa não apresentaram fragilidade; 15,1\% eram aparentemente vulneráveis; 9,1\% apresentavam fragilidade leve; e 3\%, fragilidade moderada. Entre os idosos que apresentaram algum grau de fragilidade $(n=9), 77,8 \%$ eram institucionalizados. Idosos do sexo masculino se mostraram mais frágeis (média/EFS=3,9) que os do sexo feminino (média/EFS=3,3). Indivíduos institucionalizados se mostraram mais frágeis (média/EFS=4,02) que os residentes na comunidade (média/EFS=2,69). Em termos de escolaridade, a diferença entre grupo de alfabetizados (média/EFS=3,4) e de analfabetos (média/EFS=3,6) foi relativamente pequena.

Os indivíduos que apresentaram algum grau de fragilidade (n=9) apresentaram escores médios para depressão (GDS-30) de 9 pontos. Entre os aparentemente vulneráveis ( $\mathrm{n}=5)$, a média/GDS-30 foi de 10,2 pontos; para os indivíduos que apresentaram fragilidade leve $(n=3), 10$ pontos/média/GDS-30, o que não indica prevalência de depressão entre os indivíduos vulneráveis ou com fragilidade leve. No único sujeito que apresentou fragilidade moderada, a pontuação/GDS-30 foi de 13, com indicativo de depressão leve.

O desempenho cognitivo dos sujeitos que apresentaram indicativos de fragilidade ( $\mathrm{n}=9$ ), avaliado pelo MEEM foi de 25,5 pontos (sem indicativo de declínio cognitivo) e, pelo CDR, de 1,8 pontos (tendência de demência leve a moderada). Os idosos aparentemente vulneráveis $(\mathrm{n}=5)$ apresentaram 25,8 pontos/média/MEEM e 1,6 pontos/média/CDR; os com fragilidade leve (n=3), 27,3 pontos/média/MEEM; e 1,6 pontos/média/CDR; e o idoso que 
apresentou fragilidade moderada, 19 pontos/MEEM e 3,5 pontos/CDR. O resumo dos dados pode ser observado na Tabela 1:

Tabela 1 - Resumo dos dados (idade e dos escores dos instrumentos GDS-30, EFS, MEEM e CDR) em termos de média, mediana, desvio-padrão, valor mínimo (Mínimo), valor máximo (Máximo), quartil 1 (Q1) e quartil 3 de 33 idosos residentes na comunidade e institucionalizados, Marília, SP, 2018

\begin{tabular}{|c|c|c|c|c|c|}
\hline & IDADE & GDG30 & EFS & MEEM & CDR \\
\hline $\mathrm{n}$ & 33 & 33 & 33 & 33 & 33 \\
\hline Média & 71,1 & 8,4 & 3,5 & 26,3 & 1,2 \\
\hline Mediana & 70,0 & 8,0 & 4,0 & 28,0 & 1,0 \\
\hline Desvio-Padrão & 9,0 & 3,8 & 2,4 & 3,6 & 1,1 \\
\hline Mínimo & 60,0 & 2,0 & 0 & 16,0 &, 0 \\
\hline Máximo & 93,0 & 16,0 & 10,0 & 30,0 & 4,5 \\
\hline Q1 & 64,0 & 6,0 & 1,0 & 24,5 &, 5 \\
\hline Q3 & 75,0 & 11,0 & 5,0 & 29,0 & 2,0 \\
\hline
\end{tabular}

A normalidade dos dados obtidos por meio da aplicação dos instrumentos (MEEM, CDR, GDS-30 e EFS e idade) foi verificada mediante o teste de Kolmogorov-Smirnov, com correção de Lilliefors $(\mathrm{n} \geq 30)$. Verificaram-se distribuições não normais para as distribuições dos escores do MEEM ( $\mathrm{p}=0,003)$ e CDR $(\mathrm{p}=0,001)$, e distribuições normais para as variáveis Idade $(\mathrm{p}=0,059)$, GDS30 $(\mathrm{p}=0,200)$ e Fragilidade $(\mathrm{p}=0,058)$ (Tabela 2$)$.

Tabela 2: Normalidade dos dados obtidos por meio da aplicação dos instrumentos (MEEM, CDR, GDS-30 e EFS) e idade em uma amostra de 33 idosos, Marília, SP, 2018

\begin{tabular}{lccccccc}
\hline & \multicolumn{3}{c}{ Kolmogorov-Smirnov $^{\mathrm{a}}$} & \multicolumn{3}{c}{ Shapiro-Wilk } \\
\cline { 2 - 8 } Idade & Estatística & $\mathrm{df}$ & \multicolumn{1}{c}{ Sig. } & Estatística & $\mathrm{df}$ & Sig. \\
\cline { 2 - 8 } GDG30 &, 149 & 33 & 0,059 &, 917 & 33 & 0,015 \\
EFS &, 106 & 33 & $0,200^{*}$ &, 963 & 33 & 0,311 \\
MEEM &, 150 & 33 & 0,058 &, 928 & 33 & 0,030 \\
CDR &, 195 & 33 & 0,003 &, 859 & 33 & 0,001 \\
\hline
\end{tabular}


Nesta pesquisa foi observado que a sintomatologia para depressão (GDS-30) está positivamente correlacionada com a fragilidade (EFS) e com a capacidade cognitiva (CDR).

Os resultados da análise indicam que o desempenho no MEEM influencia de modo negativo na sintomatologia para a depressão (Tabela 3). Os dados apontaram que a fragilidade influencia de maneira positiva a capacidade cognitiva. Os resultados da análise ainda indicaram correlação regular negativa entre MEEM e o CDR (Tabela 3).

Tabela 3. Correlações entre as variáveis Idade, GDS - 30, CDR, EFS e MEEM e entre resultados avaliados mediante os instrumentos (GDS-30, CDR, EFS e MEEM), de 33 idosos residentes da comunidade e institucionalizados, Marília, SP, 2018

\begin{tabular}{lcc}
\hline \multicolumn{1}{c}{ CORRELAÇÕES } & $R$ & $P$ \\
\hline Idade e GDS-30 & 0,315 & 0,074 \\
Idade e EFS & 0,117 & 0,516 \\
Idade e MEEM & $-0,477^{* *}$ & 0,005 \\
Idade e CDR & 0,295 & 0,095 \\
GDS-30 e EFS & $0,305^{*}$ & 0,046 \\
GDS-30 e CDR & $0,452^{* *}$ & 0,008 \\
GDS-30 e MEEM & $-0,577^{* *}$ & $<0,001$ \\
EFS e MEEM & $-0,257$ & 0,148 \\
EFS e CDR & $0,588^{* *}$ & $<0,001$ \\
\hline
\end{tabular}

\section{Discussão}

O objetivo desse estudo foi verificar a incidência de declínio cognitivo, depressão e fragilidade, em uma amostra composta por idosos institucionalizados e idosos residentes na comunidade e, a partir disso, identificar possíveis correlações entre essas variáveis.

A incidência de declínio cognitivo, mediante a aplicação do MEEM, só foi observada, neste estudo, em dois idosos institucionalizados, o que representa apenas $6 \%$ da amostra. Cabe ressaltar que a média de escores no MEEM foi inferior entre os idosos institucionalizados (25 contra 28,3 pontos). Entretanto, a avaliação de estados de demência/declínio cognitivo, mediante critérios do CDR, sugere que 62,6\% dos sujeitos da pesquisa apresentou indicativo para demência $(29,2 \%=$ demência leve; $27,3 \%=$ questionável; $21,2 \%$ = demência moderada; e $6 \%=$ demência grave) . 
Estudos sobre as estimativas para a prevalência de declínio cognitivo entre a população de idosos apontam uma prevalência que pode variar, considerando-se a influência do contexto sociocultural e econômico, de $7,1 \%$ a $73,1 \%{ }^{5,6}$. Estudos internacionais tais como os de Aevarsson; e Skoog (1997) e de Graves e colaboradores (1996) indicam prevalência variável entre $6,3 \%$ e $46 \%$.

Foi observada maior prevalência de indicativos de demência entre os idosos institucionalizados (3 indicativos para demência leve, 6 para moderada e, 2 indicativos para demência grave). Entre os não institucionalizados ocorreram seis indicativos, dos quais cinco para demência leve e, um para moderada. Em termos gerais, o desempenho no CDR foi inferior entre os institucionalizados, os quais obtiveram uma média 1,4, ao passo que os residentes na comunidade, 0,8 pontos. Tais resultados se mostram coerentes com Trindade e colaboradores (2013), os quais sugerem que idosos institucionalizados apresentam menor desempenho cognitivo em comparação com idosos que vivem na comunidade. Os dados relativamente contraditórios entre os resultados do MEEM e CDR podem estar relacionados ao fato de que os sujeitos da pesquisa responderam recentemente ao MEEM em outras ocasiões (avaliações relativas à anamnese ou outras pesquisas). Entretanto, é importante observar que, de acordo com Laks; Rozenghal e Engelhardt (1995), 15\% das pessoas desenvolvem inicialmente incapacidade cognitiva progressiva e, que desse total, mais ou menos 5\% dos indivíduos acima de 65 anos e $20 \%$ acima de 80 desenvolvem demência de grau moderado a grave.

Idosos alfabetizados obtiveram melhor desempenho no MEEM (27,4 pontos) do que os não-alfabetizados (21,7 pontos), o que se mostra de acordo com estudo de Soares, Coelho e Carvalho (2012) que identificaram relação estatisticamente significativa entre escolaridade, capacidade funcional e capacidade cognitiva. Tal achado também reforça a hipótese de Hall e colaboradores (2009), segundo a qual, a educação na vida inicial pode ser determinante da reserva cognitiva.

Os resultados para a prevalência de depressão apontaram que, 27,2\% dos idosos integrantes da amostra avaliada, apresentaram indicativo de depressão leve. Desse total, a maioria $(66,7 \%)$ era institucionalizada, o que confirma os dados de Heiser ${ }^{16}$ que apontam para uma prevalência de episódios depressivos em pelo menos $50 \%$ dos idosos institucionalizados, bem como os resultados de Siqueira e colaboradores (2009) que sugerem que idosos institucionalizados apresentam índices de prevalência de depressão que variam entre $15 \%$ e $50 \%$, ao passo que idosos residentes na comunidade, índices em torno de $14 \%$. 
Também se observou que, dos idosos que apresentaram indicativo de depressão, a maioria $(55,5 \%)$ era do sexo feminino, o que reforça os estudos que apontam para uma maior prevalência de episódios depressivos entre indivíduos do sexo feminino (Bertoldi, Batista, \& Ruzanowsky, 2015; Kockler, \& Heun, 2002; Angst, et al., 2002).

Foi constatado que o grupo de idosos com indicativo de depressão também apresentou, conforme resultados da avaliação mediante o CDR, tendência de demência leve a moderada (média/CDR = 1,72). Entretanto, o referido grupo, conforme avaliação feita mediante o MEEM, não apresentou indicativo de declínio cognitivo (23,9 pontos/média/MEEM). O provável motivo da disparidade entre os instrumentos foi exposto anteriormente.

De acordo com Jorm (2000), há evidências suficientes para levar a sério a possibilidade de que a depressão seja um fator de risco para demência e declínio cognitivo. Esta mesma posição é reforçada por Steffens e colaboradores (2006). Nesta pesquisa, os resultados relativos à tendência de demência nos sujeitos que apresentaram indicativo de depressão são coerentes com a premissa de Steffens e Potter (2008), segundo a qual o declínio cognitivo é comum na depressão geriátrica. Igualmente se mostra em conformidade a Ávila e Bottino (2006), cuja pesquisa indica que idosos com depressão podem apresentar maior comprometimento nas habilidades cognitivas e executivas.

A prevalência de fragilidade entre os sujeitos pesquisados foi de $27,3 \%$. O grau de fragilidade apresentado foi de aparente fragilidade em $15,1 \%$ dos sujeitos; de fragilidade leve, em $9,1 \%$ e, de fragilidade moderada em $3 \%$ dos sujeitos da pesquisa. Os idosos institucionalizados representaram $77,8 \%$ dos indivíduos que apresentaram algum grau de fragilidade. Esses sujeitos se mostraram mais frágeis $(\mathrm{EFS}=4,02)$ que os residentes em comunidade $(\mathrm{EFS}=2,69)$. Os resultados se mostram compatíveis com estudos realizados na Europa e América do Norte que apontaram que a prevalência de fragilidade varia de 5,8\% a 27,3\%, sendo maior entre idosos institucionalizados e indivíduos com idade mais avançada (Santos-Eggimann, et al., 2009; Rockwood, et al., 2004; Fried, et al., 2001). Também se mostram coerentes com estudos que indicam tendências à fragilidade que variam entre 26,7\% a 42,6\% em países em desenvolvimento (Soares, Cruz, \& Carvalho, 2016; Sousa, et al., 2012; Alvarado, et al., 2008). Neste estudo, os idosos do sexo feminino se mostraram discretamente menos frágeis $(\mathrm{EFS}=3,3)$ que os do sexo masculino $(\mathrm{EFS}=3,9)$, contrariando pesquisa de Duarte e Paúl (2015) a qual sugere que a fragilidade seja maior entre idosos do sexo feminino. 
John, Tyas e Montgomery (2013) consideram a fragilidade como fator de risco para o desenvolvimento de sintomas depressivos, ao passo que Ní Mhaoláin (2012) aponta a depressão como um dos fatores de risco para a fragilidade. Não foi identificada, nesta pesquisa, prevalência de depressão entre os sujeitos aparentemente vulneráveis ou que apresentaram fragilidade leve. Um único sujeito da pesquisa que apresentou fragilidade moderada também apresentou indicativo para depressão leve. Esses resultados não corroboram os achados de Santos e colaboradores (2014), que indicam 27,8\%, de prevalência de condição de fragilidade em idosos com indicativo de depressão; e de 51,7\% em sujeitos pré-frágeis. $\mathrm{O}$ resultado desta pesquisa sugere a necessidade de verificação da relação entre fragilidade e depressão com amostras mais amplas.

Em termos de desempenho cognitivo avaliado mediante o MEEM, os idosos que apresentaram vulnerabilidade ou de fragilidade leve não apresentaram indicativo de declínio cognitivo. O indicativo de declínio cognitivo avaliado pelo MEEM só apareceu no sujeito que apresentou fragilidade moderada (19 pontos/MEEM). Entretanto, a avaliação mediante o CDR mostrou que idosos que apresentavam algum grau de fragilidade apresentavam 1,8 de pontuação média no CDR, o que indica tendência de demência leve a moderada. Os idosos com indicativo de vulnerabilidade e os com indicativo de fragilidade leve apresentaram 1,6 pontos/média/CDR, o que aponta para um quadro de demência leve a moderada. Os resultados obtidos mediante o CDR são coerentes com os achados de Rockwood, Abeysundera e Mitnitski (2007), que identificaram a fragilidade como estando significativamente associada ao declínio cognitivo $(\mathrm{P}<0,01)$.

O cálculo das correlações entre as variáveis declínio cognitivo (MEEM e CDR), depressão (GDS-30) e fragilidade (EFS) indicaram que a depressão está positivamente correlacionada com a cognição e com a fragilidade. Ou seja, quanto melhor a saúde mental de um indivíduo, caracterizada por menor sintomatologia para depressão, menor será o declínio cognitivo e menor será a incidência de fragilidade. Os dados evidenciaram também que a probabilidade em desenvolver depressão é maior em indivíduos com declínio cognitivo, caracterizado por baixo desempenho no MEEM. Portanto, se pode considerar, assim como Ní Mhaoláin (2012), a depressão como fator de risco para a fragilidade. Também, os resultados indicam a depressão como fator de risco para declínio cognitivo, assim como já sinalizaram Jorm (2000), Steffens e colaboradores (2006), Ávila e Bottino (2006) e Steffens e Potter(2008). 
Observou-se que a fragilidade está correlacionada de maneira positiva à capacidade cognitiva. Isso significa que, quanto menos frágil um indivíduo se apresenta, melhor a sua capacidade cognitiva, o que se mostra coerente com os estudos de Luppa e colaboradores (2010), que identificaram uma forte associação entre comprometimento cognitivo e funcional. Os resultados da análise ainda indicaram correlação regular negativa entre MEEM e o CDR, o que sugere que um pior desempenho no MEEM pode estar associado a um maior grau de demência.

Esta pesquisa se mostra limitada em função do tamanho e das características da amostra. Acreditamos serem necessárias pesquisas que envolvam uma amostra maior e de diferentes características, para que se possa correlacionar, com mais precisão, as variáveis aqui analisadas, as quais se mostram de extrema complexidade.

Cabe ressaltar que o aprofundamento e a compreensão das relações entre as variáveis aqui analisadas, bem como de outras variáveis envolvidas no processo de envelhecimento, podem representar mudanças para o desenvolvimento de estratégias de intervenções direcionadas na área da saúde pública.

\section{Conclusão}

Apesar do objetivo deste estudo não ter sido o de comparar idosos institucionalizados e idosos da comunidade, é importante ressaltar que os resultados indicam que idosos institucionalizados, de forma geral, se apresentaram em piores condições, considerando-se todas as variáveis analisadas, do que os idosos residentes em comunidade. A identificação dos fatores de risco exógenos, relacionados ao declínio cognitivo, às demências, à depressão e à fragilidade em idosos, bem como as relações entre tais fatores, se mostram fundamentais para o estabelecimento de estratégias preventivas e terapêuticas. Em função disso, se faz urgente a colaboração entre pesquisadores clínicos e epidemiológicos, especialmente aqueles que trabalham nas áreas da cognição, transtornos de humor e capacidade funcional.

\section{Referências}

Aevarsson, O., \& Skoog, I. (1997). Dementia disorders in a Berth Cohort Followed from age 85 to 88: the influence of mortality refusal rate, and diagnostic change on pPrevalence. Int Psychogeriatr, 9(1), 11-23. Recuperado em 30 dezembro, 2017, de: https://www.ncbi.nlm.nih.gov/pubmed/9195275. 
Almeida, O. P. (1998). Mini-exame do estado mental e o diagnóstico de demência no Brasil. Arq. Neuro-Psiquiatr, 56(3B), 605-612. Recuperado em 30 dezembro, 2017, de: http://dx.doi.org/10.1590/S0004-282X1998000400014.

Alvarado, B. E., Zunzunegui, M. V., Beland, F., \& Bamvita, J. M. (2008). Life course social and health conditions linked to frailty in Latin American older men and women. $J$ Gerontol A Biol Sci Med Sci, 63(12), 1399-1406. Recuperado em 30 dezembro, 2017, de: https://www.ncbi.nlm.nih.gov/pubmed/19126855.

Angst, J., Gamma, A., Gastpar, M., Lépine, J. P., Mendlewicz, J., \& Tylee, A. (2002). Gender differences in depression: epidemiological findings from the European Depres I and II studies. Eur Arch Psychiatry Clin Neurosci, 252(5), 201-209. Recuperado em 30 dezembro, 2017, de: https://www.ncbi.nlm.nih.gov/pubmed/12451460.

Ávila, R., \& Bottino, C. M. C. (2006). Atualização sobre alterações cognitivas em idosos com síndrome depressiva. Rev. Bras. Psiquiatr, 28(4), 316-320. Recuperado em 30 dezembro, 2017, de: http://dx.doi.org/10.1590/S1516-44462006005000010.

Bernard, C. (1878/1996). Leçons sur les phénomènes de la vie communs aux aniamux et aux végétaux. Paris, France: Vrin (1 $1^{\mathrm{a}}$ ed., Paris: Baillière, 1878).

Bertoldi, J. T., Batista, A. C., \& Ruzanowsky, S. (2015). Declínio cognitivo em idosos institucionalizados: revisão de literatura. Rev Dep Educ Fís Saúde Mest Promoç Saúde Univ. Santa Cruz Sul, 16(2), 152-156. Recuperado em 30 dezembro, 2017, de: file:///C:/Users/Dados/Downloads/5411-28653-1-PB.pdf.

Bertolucci, P. H., Okamoto, I. H., Brucki, S. M., \& Ramos, I. R. (2001). Applicability of the CERAD neuropsychological battery to Brazilian elderly. Arq Neuropsiquiatr, 59(3a), 532536. Recuperado em 30 dezembro, 2017, de: http://dx.doi.org/10.1590/S0004282X2001000400009.

Callegari-Jacques, S. M. (2003). Bioestatística: princípios e aplicações. Porto Alegre, RS: Artmed.

Cruz, D. T., Cruz, F. M., Ribeiro, A. L., Veiga, C. L., \& Leite, I. C. G. (2015). Associação entre capacidade cognitiva e ocorrência de quedas em idosos. Cad. Saúde Colet., 23(4), 386393. Recuperado em 30 dezembro, 2017, de: http://www.scielo.br/pdf/cadsc/v23n4/1414462X-cadsc-23-4-386.pdf.

Duarte, M., \& Paúl, C. (2015). Prevalência de fragilidade fenotípica em pessoas em processo de envelhecimento numa comunidade portuguesa. Rev Bras Geriatr Gerontol, 18(4), 871880. Recuperado em 30 dezembro, 2017, de: http://dx.doi.org/10.1590/18099823.2015.14160.

Duron, E., \& Hanon, O. (2008). Hypertension, cognitive decline and dementia. Arch Cardiovasc Dis., 101(3), 181-189. Recuperado em 30 dezembro, 2017, de: https://www.ncbi.nlm.nih.gov/pubmed/18477946.

Fabrício-Wehbe, S. C. C., Schiaveto, F. V., Vendrusculo, T. R. P., Haas, V. J., Dantas, R. A. S., \& Rodrigues, R. A. P. (2009). Cross-cultural adaptation and validity of the "Edmonton Frail Scale - EFS" in a Brazilian elderly sample. Rev. Latino-Am. Enfermagem, 17(6), 10431049. Recuperado em 30 dezembro, 2017, de: http://dx.doi.org/10.1590/S010411692009000600018.

Folstein, M. F., Folstein, S.E \& Mchugh, P.R. (1975). Mini-Mental State: a practical method for grading the cognitive state of patients for the clinician. J Psychiatr Res., 12(3), 189-198. Recuperado em 30 dezembro, 2017, de: https://www.ncbi.nlm.nih.gov/pubmed/1202204. 
Fried, L. P., Tangen, C. M., Walston, J., Newman, A. B., Hirsch, C., Gottdiener, J., Seeman, T., Tracy, R., Kop, W. J., Burke, G., \& McBurnie, M. A. (2001). Frailty in older adults: evidence for a phenotype. J Gerontol Ser A Biol Sci Med Sci., 56(3), 146-156. Recuperado em 30 dezembro, 2017, de: https://www.ncbi.nlm.nih.gov/pubmed/11253156.

Gobbens, R. J. J., Luijkx, K. G., Wijnen-Sponselee, M. T., \& Schols, J. M. G. A. (2010). In search of an integral conceptual definition of frailty: opinions of experts. J Am Med Dir Assoc., 11(5), 338-s43. Recuperado em 30 dezembro, 2017, de: doi: 10.1016/j.jamda.2009.09.015.

Graves, A. B., Larson, E. B., Edland, S. D., Bowen, J. D., McCormick, W. C., McCurry, S. M., Rice, M. M., Wenzlow, A., \& Uomoto, J. M. (1996). Prevalence of dementia and its subtypes in the Japanese American population of king country, Washington state. The Kame Project. Am J. Epidemiol., 144(8), 760-771. Recuperado em 30 dezembro, 2017, de: https://www.ncbi.nlm.nih.gov/pubmed/8857825.

Gurian, M. B. F., De Oliveira, R. C., Laprega, M. R., \& Rodrigues Júnior, A. L. (2012). Rastreamento da função cognitiva de idosos não-institucionalizados. Rev Bras Geriatr Gerontol., 15(2), 275-284. Recuperado em 30 dezembro, 2017, de: http://dx.doi.org/10.1590/S1809-98232012000200010.

Gurland, B., Kuriansky, J., Sharpe, L., \& Simon R. (1978). The comprehensive assessment and referral evaluation (care): rationale, development and reliability. Int. J. Aging hum. Develop., $\quad 8(1), 9-42 . \quad$ Recuperado em 30 dezembro, 2017, de: https://www.ncbi.nlm.nih.gov/pubmed/873639.

Hall, C. B., Lipton, R. B., Sliwinski, M., Katz, M. J., Derby, C. A., \& Verghese, J. (2009). Cognitive activities delay onset of memory decline in persons who develop dementia. Neurology, 73(5), 356-361. Recuperado em 30 dezembro, 2017, de: doi: 10.1212/WNL.0b013e3181b04ae3.

Heiser, D. (2004). Depression Identification in the Long-Term Care Setting. Clinical Gerontologist, 27(4), 3-18. Recuperado em 30 dezembro, 2017, de: https://www.tandfonline.com/doi/abs/10.1300/J018v27n04_02.

Hernandéz, S. P. S., \& Mocetzuma, L. (1999). Depresión en población adulta mayor: tamizage en unidade de primer nivel de atención médica. Rev Med IMSS., 37(2), 111-115. Recuperado em 30 dezembro, 2017, de: http://bases.bireme.br/cgibin/wxislind.exe/iah/online/?IsisScript=iah/iah.xis\&src=google \&base=LILACS\&lang=p\&ne xtAction=lnk\&exprSearch=266763\&indexSearch=ID.

Herrera, J. E., Caramelli, P., \& Nitrini, R. (1998). Estudo epidemiológico populacional de demência na cidade de Catanduva. Rev Psiquiatr Clín., 25(2), 70-73. Recuperado em 30 dezembro, 2017, de: https://scholar.google.com.br/citations?user=oYGhFVsAAAAJ\&hl=ptBR\#d=gs_md_cita-d\&u=\%2Fcitations\%3Fview_op\%3Dview_citation\%26hl\%3DptBR\%26user\%3DoYGhFVsAAAAJ\%26citation_for_view\%3DoYGhFVsAAAAJ\%3A2osOg NQ5qMEC\%26tzom\%3D180.

Hughes, C. P., Berg, L., Danziger, W. L., Coben, L. A., \& Martin, R. L. (1982). A new clinical scale for the staging of dementia. Br J Psychiatry, 140, 566-572. Recuperado em 30 dezembro, 2017, de: doi: 10.1192/bjp.140.6.566.

John, P. D. S., Tyas, S. L., \& Montgomery, P. R. (2013). Depressive symptoms and frailty. Int J Geriatr Psychiatry, 28(6), 607-614. Recuperado em 30 dezembro, 2017, de: https://doi.org/10.1002/gps.3866. 
Jorm, A. F. (2000). Is Depression a Risk Factor for Dementia or Cognitive Decline? Gerontology, 46, 219-227. Recuperado em 30 dezembro, 2017, de: https://www.ncbi.nlm.nih.gov/pubmed/10859462.

Kockler, M., \& Heun, R. (2002). Gender differences of depressive syntoms in depressed and non depressed elderly persons. Int J Geriatr Psychiatry, 17(1), 65-72. Recuperado em 30 dezembro, 2017, de: https://www.ncbi.nlm.nih.gov/pubmed/11802233.

Laks, J., Rozenghal, M., \& Engelhardt, E. (1995). Sintomas psiquiátricos na doença de Alzheimer e sua relação com o estado cognitivo. Rev Bras Neurol Psiquiatr, 31(5), 225-234. Recuperado em 30 dezembro, 2017, de: http://bases.bireme.br/cgibin/wxislind.exe/iah/online/?IsisScript=iah/iah. $x i s \& s r c=$ google \&base=LILACS\&lang=p\&ne xtAction=lnk\&exprSearch=166863\&indexSearch=ID.

Lindesay, J., Briggs, K., \& Murphy, E. (1989). The Guy's Age Concern Survey: prevalence rates of cognitive impairment, depression and ansiety in a urban elderly community. Brit. $J$. Psychiat., 155, 317-329. Recuperado em 30 dezembro, 2017, de: https://www.ncbi.nlm.nih.gov/pubmed/2611541.

Montaño, M. B. M. M., \& Ramos, L.R. (2005). Validade da versão em português da Clinical Dementia Rating. Rev. Saúde Pública, 39(6), 917-927. Recuperado em 30 dezembro, 2017, de: http://www.scielo.br/pdf/rsp/v39n6/26985.pdf.

Morris, J. (1993). The Clinical Dementia Rating (CDR): current version and scoring rules. Neurology, 43(11), 2412-2414. Recuperado em 30 dezembro, 2017, de: https://n.neurology.org/content/43/11/2412.2.

Ní Mhaoláin, A. M., Fan, C. W., Romero-Ortuno, R., Cogan, L., Cunningham, C., Kenny, R. A., \& Lawlor, B. (2012). Frailty, depression, and anxiety in later life. Int Psychogeriatr, 24(8), 1265-1274. Recuperado em 30 dezembro, 2017, de: doi: 10.1017/S1041610211002110.

Rabelo, D. F. (2009) Declínio cognitivo leve em idosos: fatores associados, avaliação e intervenção. Rev Min Ciênc Saúde, 1(1), 56-68. Recuperado em 30 dezembro, 2017, de: https://repositorio.ufscar.br/bitstream/handle/ufscar/9415/DissFGC.pdf?sequence=1\&isAllowed=y.

Rockwood, K., Abeysundera, M. J., \& Mitnitski, A. (2007). How should we grade frailty in nursing home patients? J Am Med Dir Assoc., 8(9), 595-603. Recuperado em 30 dezembro, 2017, de: https://www.ncbi.nlm.nih.gov/pubmed/17998116.

Rockwood, K., Howlett, S. E., MacKnight, C., Beattie, B. L., Bergman, H., Hebert, R., Hogan, D. B., Wolfson, C., \& McDowell, I. (2004). Prevalence, attributes, and outcomes of fitness and frailty in community dwelling older adults: report from the Canadian study of health and aging. J Gerontol A Biol Sci Med Sci., 59(12), 1310-1317. Recuperado em 30 dezembro, 2017, de: https://www.ncbi.nlm.nih.gov/pubmed/15699531.

Rolfson, D. B., Majumdar, S. R., Tsuyuki, R. T., Tahir, A., \& Rockwood, K. (2006). Validity and reliability of the Edmonton Frail Scale. Age Ageing, 35(5), 526-529. Recuperado em 30 dezembro, 2017, de: doi: 10.1093/ageing/af1041.

Rozenthal, M., Laks, J., \& Engelhardt, E. (2004). Neuropsychological aspects of depression. R. Psiquiatr., 26(2), 204-212. Recuperado em 30 dezembro, 2017, de: http://dx.doi.org/10.1590/S0101-81082004000200010. 
Santos, D. M. T., Almeida, E. G., Santos Ferreira, P. C., Dias, F. A., \& Pegorari, M. S. (2014). Status de fragilidade entre idosos com indicativo de depressão segundo o sexo. J Bras Psiquiatr, 63(4), 347-353. Recuperado em 30 dezembro, 2017, de: http://www.scielo.br/pdf/jbpsiq/v63n4/0047-2085-jbpsiq-63-4-0347.pdf.

Santos-Eggimann, B., Cuenoud, P., Spagnoli, J., \& Junod, J. (2009). Prevalence of frailty in middle-aged and older com - munity-dwelling Europeans living in 10 countries. $J$ Gerontol A Biol Sci Med Sci., 64(6), 675-681. Recuperado em 30 dezembro, 2017, de: doi: 10.1093/gerona/glp012.

Soares, E., Coelho, M. O., \& Carvalho, S. M. (2012). Capacidade funcional, declínio cognitivo e depressão em idosos institucionalizados: possibilidade de relações e correlações. Revista Kairós-Gerontologia, 15(5), 117-139. Recuperado em 30 dezembro, 2017, de: https://revistas.pucsp.br/index.php/kairos/article/view/9541.

Soares, E., Cruz, A. E. B., \& Carvalho, S. M. R. (2016). Qualidade de vida, síndrome de fragilidade e declínio cognitivo em idosos institucionalizados. Revista Kairós-Gerontologia, 19(4), 223-241. Recuperado em 30 dezembro, 2017, de: https://revistas.pucsp.br/kairos/article/view/33231.

Siqueira, G. R., Vasconcelos, D. T., Duarte, G. C., Arruda, I. C., Costa, J. A. S., \& Cardoso, R. O. (2009) Análise da sintomatologia depressiva nos moradores do Abrigo Cristo Redentor através da aplicação da Escala de Depressão Geriátrica (EDG). Ciên Saúde Colet., 14(1), 253-259. Recuperado em 30 dezembro, 2017, de: http://dx.doi.org/10.1590/S141381232009000100031.

Sousa, A. C., Dias, R. C., Maciel, A. C., \& Guerra, R. O. (2012). Frailty syndrome and associated factors in communitydwelling elderly in Northeast Brazil. Arch Gerontol Geriatr., 54(2), 95-101. Recuperado em 30 dezembro, 2017, de: doi: 10.1016/j.archger.2011.08.010.

Steffens, D. C., Otey, E., Alexopoulos, G. S., Butters, M. A., Cuthbert, B., Ganguli, M., Geda, Y. E., Hendrie, H. C., Krishnan, R. R., Kumar, A., Lopez, O. L., Lyketsos, C. G., Mast, B. T., Morris, J. C., Norton, M. C., Peavy, G. M., Petersen, R. C., Reynolds, C. F., Salloway, S., Welsh-Bohmer, K. A, \& Yesavage, J. (2006). Perspectives on Depression, Mild Cognitive Impairment, and Cognitive Decline. Arch Gen Psychiatry, 63(2). 130-138. Recuperado em 30 dezembro, 2017, de: https://www.ncbi.nlm.nih.gov/pubmed/16461855.

Steffens, D. C., \& Potter, G. G. (2008). Geriatric depression and cognitive impairment. Psychol Med., 38(2), 163-175. Recuperado em 30 dezembro, 2017, de: https://www.ncbi.nlm.nih.gov/pubmed/17588275.

Stoppe Júnior, A., Jacob Filho, W., \& Louzã Neto, M. R. (1994). Avaliação de Depressão em Idosos através da Escala de Depressão em Geriatria: resultados preliminares. Revista da ABPAPAL, 16(4), 149-153. Recuperado em 30 dezembro, 2017, de: http://pepsic.bvsalud.org/scieloOrg/php/reflinks.php?refpid=S1677-0471200600010000900042\&pid= S1677-04712006000100009\&lng=pt.

Trichard, C., Martinot, J. L., Alagille, M., Masure, M. C., Hardy, P., Ginestet, D., \& Feline, A. (1995). Time course of prefrontal lobe dysfunction in severely depressed in patients: a longitudinal neuropsychological study. Psychol Med., 25(1), 79-85. Recuperado em 30 dezembro, 2017, de: https://www.ncbi.nlm.nih.gov/pubmed/7792365.

Trindade, A. P. N. T., Barboza, M. A., De Oliveira, F. B., \& Borges, A. P. O. (2013). Repercussão do declínio cognitivo na capacidade funcional em idosos institucionalizados e não institucionalizados. Fisioter Mov, 26(2), 281-289. Recuperado em 30 dezembro, 2017, de: http://dx.doi.org/10.1590/S0103-51502013000200005. 
Yesavage, J. A., \& Sheikh, J. I. (1986). Geriatric depression scale (GDS) recent evidence and development of a shorter version. Clin Gerontol, 5(1-2), 165-173. Recuperado em 30 dezembro, 2017, de: https://doi.org/10.1300/J018v05n01_09.

Recebido em 04/12/2018

Aceito em 31/12/2018

\begin{abstract}
Aléxia Ximenes Torqueti - Fisioterapeuta, especialista em Fisioterapia em Terapia Intensiva Adulto, Pediátrico e Neonatal. Universidade Estadual Paulista, Unesp. Centro de Estudos da Educação e da Saúde, CEES. Faculdade de Filosofia e Ciências, Universidade Estadual Paulista, UNESP.

E-mail: ximenesta@hotmail.com
\end{abstract}

Edvaldo Soares - Doutor em Neurociências. Coordenador do Laboratório de Neurociência Cognitiva, LaNeC, Faculdade de Filosofia e Ciências, FFC, Unesp, Campus de Marília, SP. ORCID iD: https://orcid.org/0000-0003-3021-669X

E-mail: edvaldo.soares@unesp.br 